\title{
Efeito da Adição de Diferentes Copolímeros em Blendas HDPE/HIPS Pós-Consumo: Morfologia de Fases e Propriedades Térmicas
}

\author{
Igor S. B. Perez, Sati Manrich, Silvio Manrich \\ Departamento de Engenharia de Materiais, 3R-nrr, UFSCar
}

\begin{abstract}
Resumo: Blendas de poliolefinas/HIPS têm sido exploradas para obter filmes especiais com determinadas propriedades desejadas, tornando imperativo desenvolver vários estudos para um melhor conhecimento do comportamento desses materiais. Neste trabalho, efeitos da adição dos copolímeros comerciais de estireno-butadieno multibloco (SBS) e de estireno-(etilenoco-butileno)-estireno (SEBS) tribloco linear em blendas pós-consumo de HDPE e HIPS são reportados. A diminuição nas dimensões da microfase dispersa, aliada à rugosidade superficial da fase HDPE após extração seletiva do HIPS, independentemente de a fase matriz ser HIPS ou HDPE, mostraram mais eficiência do SEBS como modificador interfacial de tensão ou como surfactante entre os diferentes domínios quando comparado com o SBS. Os resultados das caracterizações térmicas, por exemplo, menor $\mathrm{T}_{\mathrm{m}}$ e menor grau de cristalinidade do HDPE, e maior $\mathrm{T}_{\mathrm{g}}$ do poliestireno na presença de SEBS corroboraram esta conclusão, como será discutido posteriormente.
\end{abstract}

Palavras-chave: HDPE/HIPS pós-consumo, blendas, reciclagem, compatibilização, morfologia, caracterização térmica.

\section{The Effect of Different Block Copolymers on Post Consumer HDPE/HIPS Blends: Phase Morphology and Thermal Properties}

Abstract: Blending of post-consumer polyolefins/HIPS has been exploited for obtaining special films with a desired set of properties, which has required studies to understand the behavior of these materials. In this work the effects of commercial multiblock styrene-butadiene (SBS) and linear triblock styrene-(ethylene-co-butylene)-styrene (SEBS) copolymers in blends of post-consumer high density polyethylene (HDPE) and HIPS are reported. Thermal properties and phase morphology were comparatively analyzed for the additives aiming at verifying possible correlations between them. Decreased dimensions of the minor micro phase along with HDPE surface roughness after HIPS selective extraction, independently of the matrix being HIPS or HDPE, showed better effectiveness for SEBS as interfacial tension modifier or as surfactant at the different domains interface when compared with SBS. The results of thermal characterizations, e.g. lower HDPE melting temperature, lower degree of crystallinity, and higher polystyrene glass transition temperature in the presence of SEBS corroborated with this conclusion as it will be discussed further.

Keywords: Post consumer HDPE/HIPS, blends, recycling, compatibilization, morphology, thermal characterization.

\section{Introdução}

A prática da reciclagem de matérias primas previamente utilizadas já está consolidada mundialmente como contribuição imprescindível ao desenvolvimento sustentável. No Brasil, 16,5\% dos plásticos rígidos e filmes são reciclados, o que equivale a cerca de 200 mil toneladas por ano. A taxa de reciclagem de plástico na Europa há anos está estabilizada em $22 \%$, sendo que em alguns países a prática é impositiva e regulada por legislações complexas e custosas para a população local, diferentemente do Brasil, onde a reciclagem acontece de forma espontânea. O Brasil ocupa o $4^{\circ}$ lugar na reciclagem mecânica do plástico, ficando atrás apenas da Alemanha, Áustria e EUA ${ }^{[1,2]}$.

Na reciclagem mecânica, produtos de alto valor agregado fabricados com os resíduos são mais competitivos de forma a ampliar o mercado desses polímeros reciclados.
Para viabilizar a obtenção de tais produtos, deve-se assegurar eficiência no processo de separação quando polímeros individuais são utilizados, ou eficiência na mistura de polímeros quando diferentes fases que constituem a base polimérica possam atender o desempenho desejado, o que torna imprescindível o conhecimento aprofundado do comportamento das misturas. No sentido de obter controle, soma e até sinergia de propriedades, blendas binárias e ternárias têm sido amplamente pesquisadas utilizando-se diferentes aditivos e métodos de compatibilização. Dentre elas, as de poliolefinas com PS e HIPS podem ser destacadas ${ }^{[2-14]}$.

Para compatibilização, copolímeros em bloco com variados comonômeros, composição, peso molecular e estrutura, em conjunto ou não com processos reativos, são os mais estudados. Resultados interessantes de estabilização da morfologia de fases em blendas PS/HDPE, nas proporções 90/10 e 80/20\% em peso, foram obtidos por Tao et al. ${ }^{[15]}$. 
Estes concluíram que o processo conhecido como pulverização por cisalhamento no estado sólido SSSP - solid state shear pulverization, comparado com processos de mistura de blendas por fusão, é muito mais eficiente para compatibilizar os dois polímeros ao utilizar SEBS como compatibilizante.

Chen et al. ${ }^{[16]}$ observaram que a morfologia de fases de blendas HDPE/PS sindiotático (sPS) 80/20 em peso foi alterada, tendo reduzido significativamente as dimensões da fase dispersa sPS com a adição de compatibilizantes SEBS tribloco e estireno-b-(etileno-co-butileno) (SEB) dibloco. Ocorreu pequena diminuição na temperatura de fusão $\mathrm{T}_{\mathrm{m}}$ das duas fases e no grau de cristalinidade $(x)$, entretanto ocorreu redução significativa em $x_{\mathrm{c}}$ do HDPE, com o aumento da adição dos copolímeros. Isto foi atribuído à penetração das moléculas de copolímero na interface das interfases, mas também à dispersão destes na fase matriz HDPE quando em altas concentrações, agindo então como diluente polimérico.

Apesar do grande número de publicações sobre compatibilização de blendas de poliolefinas com PS ${ }^{[3-5,7-12,14]}$, foram muito raros os estudos encontrados sobre pares poliolefinas/ HIPS ${ }^{[6,13]}$. Entretanto, o poliestireno de alto impacto e o expandido (EPS) estão entre os materiais com maior participação na composição dos resíduos plásticos urbanos, ao contrário do poliestireno homopolímero PS, de forma que estudos com HIPS são importantes quando se trata da reciclagem de poliestireno pós-consumo.

Considerando uma pesquisa mais ampla sobre filmes especiais obtidos com plásticos de resíduo urbano desenvolvida por este Grupo, blendas de poliolefinas e HIPS pós-consumo foram estudadas quanto ao efeito da adição de agentes compatibilizantes sobre as propriedades das blendas e dos filmes obtidos com as mesmas ${ }^{[2,17-19]}$. No presente trabalho, foram realizados estudos comparativos do efeito da adição de copolímeros multibloco SBS e linear SEBS, especificamente em blendas de HDPE e HIPS pós-consumo. Foram analisadas as variações em propriedades térmicas e em morfologia de fases decorrentes da influência dos fatores citados, verificando-se as correlações existentes entre as mesmas.

\section{Experimental}

Os resíduos pós-consumo de HIPS e HDPE foram coletados na central de resíduos da UFSCar, apresentando índices de fluidez, 4,98 g/10 minutos (peso de 5,0 kg) e $0,28 \mathrm{~g} / 10$ minutos (peso de 2,16 kg), respectivamente. $\mathrm{O}$ procedimento para a recuperação mecânica da matéria prima foi o convencional descrito em publicações anteriores do grupo $^{[2,18,19]}$. Os agentes compatibilizantes comerciais SEBS Kraton® G e SBS Styrolux ${ }^{\circledR}$ 684D, são da Kraton e da Basf, respectivamente. Segundo os catálogos eletrônicos das empresas, o SEBS é tribloco linear e o SBS é multibloco na forma de estrela, porém especificações da massa molar de cada bloco em ambos os copolímeros ou do número de blocos para o SBS não são fornecidas. Dados informais obtidos pessoalmente indicaram que o SBS tenha quatro braços, en- tretanto caracterizações a serem ainda realizadas fornecerão resultados mais confiáveis para trabalhos futuros.

As composições HDPE/HIPS estudadas foram 100/0, 80/20, 60/40, 40/60, 20/80 e 0/100 sem e com copolímero, o qual foi fixado em $7,5 \%$, baseado em resultados obtidos em trabalhos anteriores ${ }^{[17-19]}$. Para a blenda HDPE/HIPS, na referência ${ }^{[19]}$, os resultados foram melhores com $10 \%$ em peso de copolímero do que com 5\%, entretanto não se analisou uma concentração intermediária, o que justificou adição de 7,5\% no presente trabalho. As blendas compatibilizadas foram referidas pela composição inicial HDPE/HIPS, entretanto considerando a mistura ternária HDPE/HIPS/copolímero, as concentrações $100,80,60,40$ e $20 \%$ foram na realidade 92,$5 ; 74 ; 55,5 ; 37$ e $18,5 \%$ respectivamente e $7,5 \%$ para os compatibilizantes.

O método para misturar os componentes foi o de "duas etapas" ${ }^{20]}$, onde se fez pré-mistura HDPE/HIPS, e mistura final adicionando-se o componente compatibilizante. O perfil de temperaturas na extrusora mono-rosca Gerst para as duas etapas foi 170,190 e $220{ }^{\circ} \mathrm{C}$, com tempo de residência de aproximadamente 2 minutos. Como o objetivo era submeter as amostras às mesmas condições de tempo e temperatura na extrusora Gerst, esse controle foi feito com pequenas variações na rotação da rosca que não foi registrada. As blendas sem agente compatibilizante também foram submetidas à segunda etapa, visando a uma condição reproduzida para todas as amostras.

A morfologia de fases foi caracterizada segundo o procedimento descrito por $\mathrm{Wu}$ et al. ${ }^{[20]}$, analisando-se superfícies fraturadas criogenicamente, em que tolueno foi usado como solvente para extração seletiva de HIPS e copolímeros SBS e SEBS, por 48 horas à temperatura ambiente. Após a extração, as amostras foram secas a $70{ }^{\circ} \mathrm{C}$ por 24 horas, antes de recobrimento com ouro e observação no microscópio Philips XL30 Field Emission Gun (FEG). As caracterizações térmicas foram realizadas por calorimetria diferencial, DSC modelo Q100, da TA Instruments, em atmosfera inerte de $\mathrm{N}_{2}$ super-seco. Foi feito um aquecimento à taxa de $10^{\circ} \mathrm{C} / \mathrm{min}$ até $150^{\circ} \mathrm{C}$, deixando-se a amostra fundir e estabilizar a essa temperatura por dois minutos, seguido de resfriamento controlado a uma taxa de $20^{\circ} \mathrm{C} / \mathrm{min}$ e aquecimento final a uma taxa de $10{ }^{\circ} \mathrm{C} / \mathrm{min}$. A entalpia de fusão do HDPE $100 \%$ cristalino foi considerada $293 \mathrm{~J} \cdot \mathrm{g}^{-1[14]}$.

\section{Resultados e Discussão}

As micrografias obtidas por SEM/FEG foram primeiramente analisadas para as blendas HDPE/HIPS 80/20, 60/40, $40 / 60$ e 20/80, \% em peso, não compatibilizadas, as quais são mostradas na Figura 1. As amostras não compatibilizadas apresentaram características típicas de blendas imiscíveis compostas por um componente cristalino e um amorfo ${ }^{[3,4]}$. Os domínios esféricos da fase dispersa de HIPS (Figuras 1a, b), com superfícies bem lisas, evidenciaram praticamente nenhuma adesão entre as fases. 


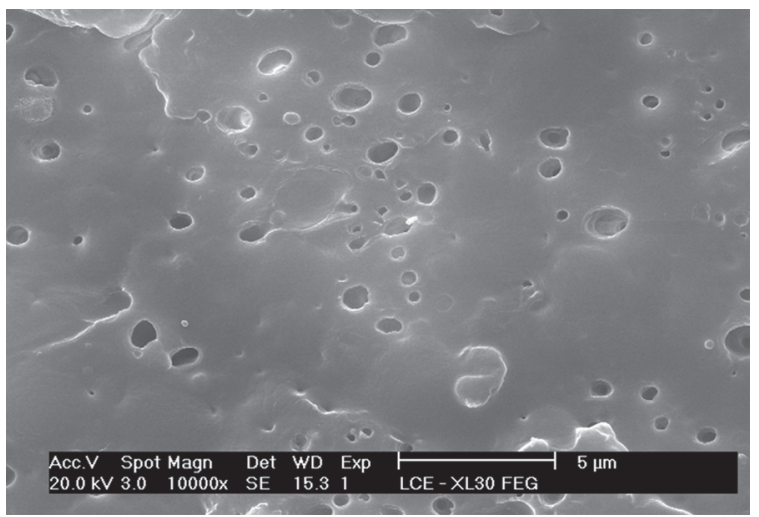

(a)

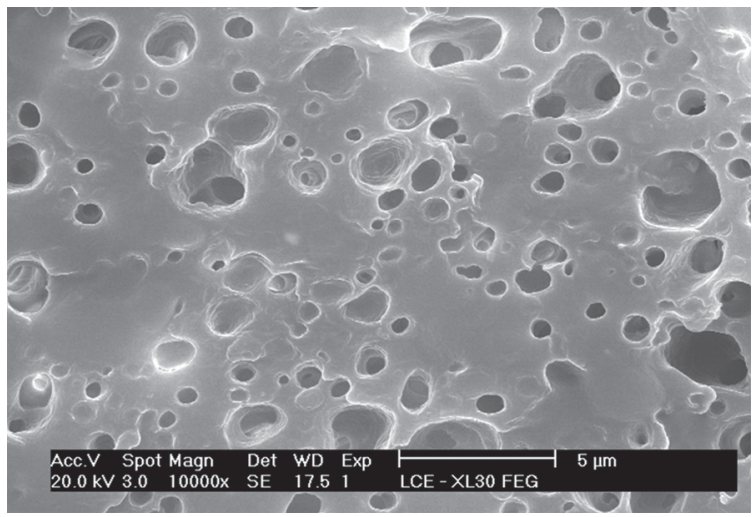

(b)

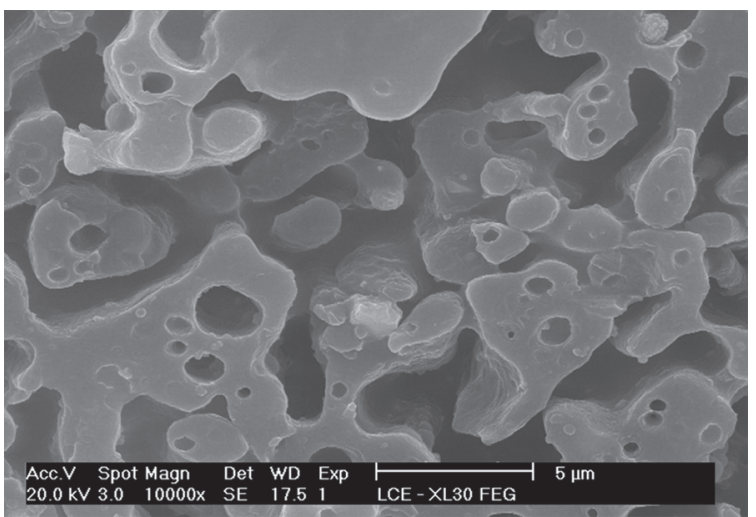

(c)

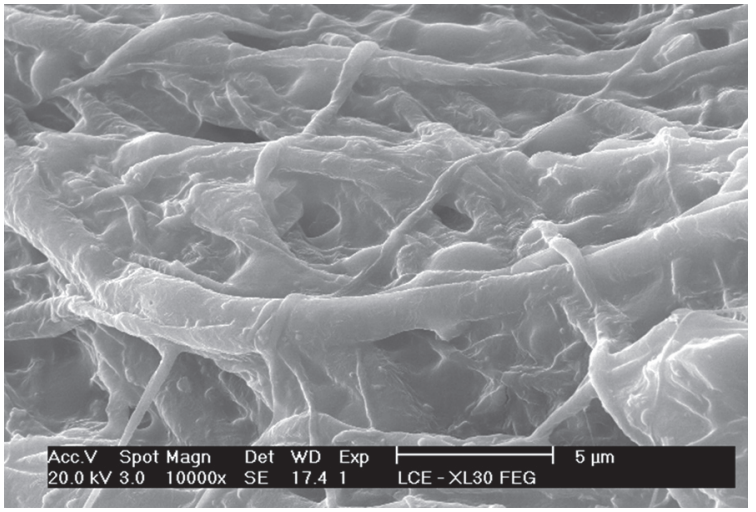

(d)

Figura 1. Micrografias em MEV/FEG das amostras de HDPE/HIPS sem compatibilizante a) 80/20; b) 60/40; c) 40/60; e d) 20/80. Barra dimensio$\mathrm{nal}=5 \mu \mathrm{m}$.

Aumentando-se a quantidade de HIPS para $60 \%$ em peso (Figura 1c), a morfologia mostra que já ocorreu a inversão de fases, em que, além de se terem ainda presentes pequenos domínios de HIPS dispersos no HDPE, formou-se um caminho contínuo da nova fase matriz HIPS ao longo de toda a amostra. A inversão de fases é um fenômeno muito comum em blendas imiscíveis, que depende de vários fatores como características reológicas e interfaciais dos componentes, das condições de processamento, entre outros. É possível estimar a região de inversão para pares poliméricos, tendo-se as razões de viscosidades do fundido e das frações volumétricas dos componentes, desde que a baixas taxas de cisalhamento e não considerando variações de tensão interfacial entre as fases $^{[3,4]}$. Essa estimativa e a sua análise fogem ao escopo do presente trabalho, tendo sido incluídas para futuras publicações.

Comparando as micrografias $1 \mathrm{~b}$ e $1 \mathrm{c}$, é interessante observar que a morfologia da blenda com $60 \%$ de HIPS assemelha-se à morfologia co-contínua mais do que a da blenda com $40 \%$ de HIPS. Isto provavelmente indique que as concentrações da região de inversão de fases estejam mais próximas à da blenda HDPE/HIPS 40/60 do que à da 60/40[3,4,7-9], entretanto, uma conclusão definitiva a respeito só será possível após análise das suas características reológicas.

A micrografia 1d corresponde à blenda HDPE/HIPS (20/80) não compatibilizada. Observa-se claramente uma fase dispersa HDPE em forma de fibras, que restou em meio a espaços antes ocupados pela fase matriz contínua de HIPS extraída com tolueno. O diâmetro das fibras varia de 0,5 a $3 \mu \mathrm{m}$, porém o seu comprimento é substancial. Na extensa bibliografia consultada, verificou-se que essa morfologia de fases foi divulgada unicamente por $\mathrm{Wu}$ e colaboradores ${ }^{[20]}$, também para blendas HDPE/PS 20/80, em que as longas fibras apresentaram diâmetro entre 0,5 e $5 \mu \mathrm{m}$. Consideraram que tal comprimento das fibras denotava que o tamanho das gotas de HDPE havia sido bastante grande antes de serem convertidas em fibras durante o processo de extrusão, o que por sua vez indicou pobre interação entre a fase dispersa e a matriz. A superfície lisa e "limpa" da fase fibrosa de HDPE, após a extração de HIPS, evidencia a incompatibilidade, da mesma forma que verificada por Wu et al. ${ }^{[20]}$.

$O$ efeito dos modificadores interfaciais na morfologia das blendas com 20, 40 e 60\% de HIPS está representado nas micrografias da Figura 2. Para essas amostras, a adição tanto de SBS quanto de SEBS ocasionou uma diminuição do tamanho médio dos domínios de HIPS, como esperado, e as superfícies dos domínios de HDPE, quando comparadas às das blendas não compatibilizadas, exibem maior rugosidade tanto na região de fratura quanto nas cavidades originadas pela extração do HIPS. Isso é devido ao fato de os agentes compatibilizantes penetrarem nas diferentes fases e de serem também solúveis em tolueno, deixando portanto vazios os es- 
paços antes ocupados pelos segmentos miscíveis ou compatíveis com HDPE após sua dissolução ${ }^{[20]}$. E da mesma forma que as correspondentes não compatibilizadas, a morfologia considerada mais semelhante à da co-contínua foi observada somente para a composição com $60 \%$ de HIPS dentre as analisadas.

Comparando as superfícies das blendas quanto ao tipo de compatibilizante utilizado, é possível observar que o SEBS proporcionou aos domínios de HIPS tamanhos menores e melhor distribuídos, o que indicou que o poder de compatibilização do copolímero SEBS tribloco linear foi maior quando comparado ao SBS multibloco. Especificamente para a

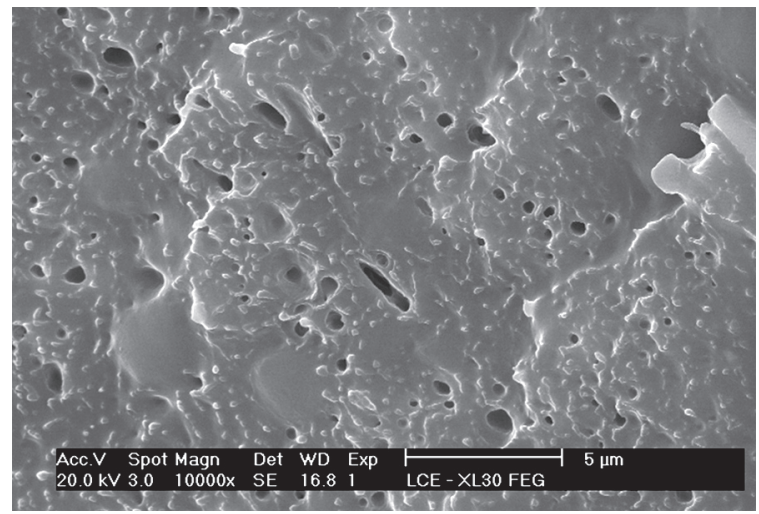

(a)

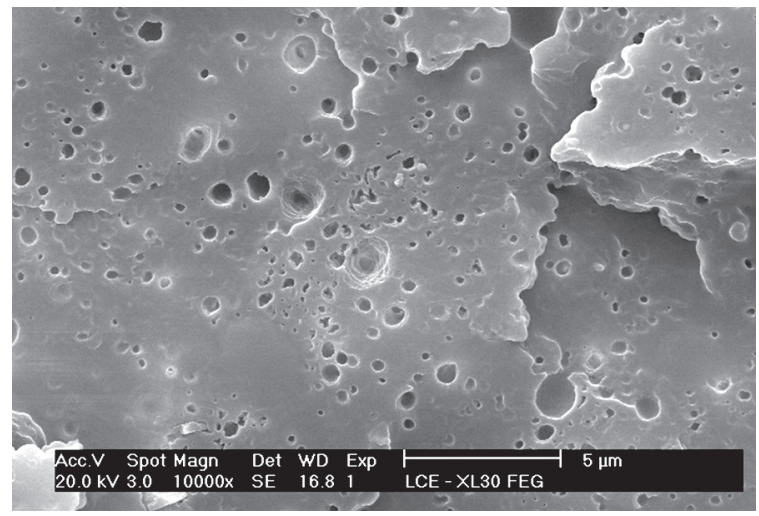

(b)

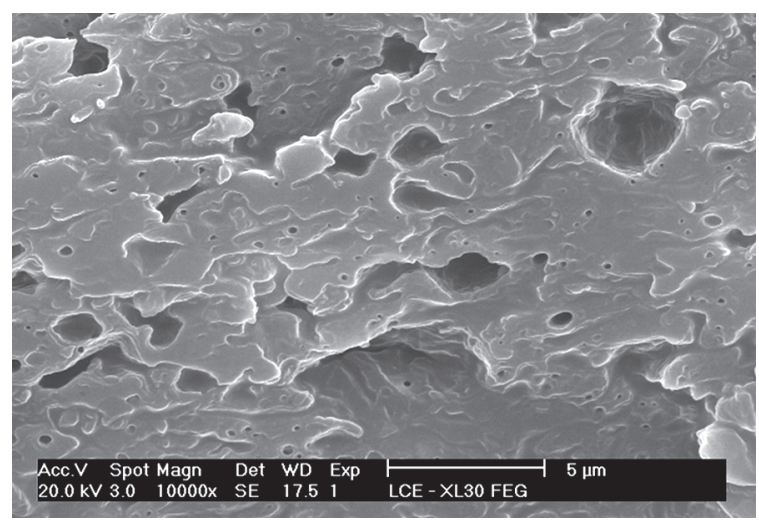

(c) morfologia das blendas com 60\% de HIPS, Figuras 2e e 2f, a rede formada pela interpenetração entre as fases é visivelmente mais intrincada e de tamanhos menores, evidenciando uma maior eficiência do SEBS quando comparado ao SBS. Provavelmente, isto seja decorrente de uma maior facilidade de a estrutura linear do copolímero tribloco em interagir e até se misturar simultaneamente às diferentes fases, além de os segmentos de etileno-butileno do SEBS serem mais compatíveis com HDPE do que os de butadieno do SBS.

Ao contrário do que se esperava e do que foi obtido na referência ${ }^{[20]}$, as amostras de blendas HDPE/HIPS 20/80 compatibilizadas não apresentaram a mesma morfologia fi-

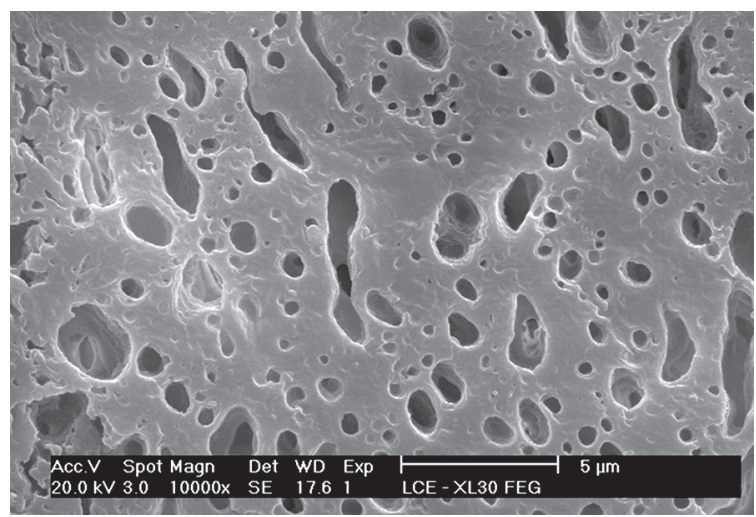

(d)

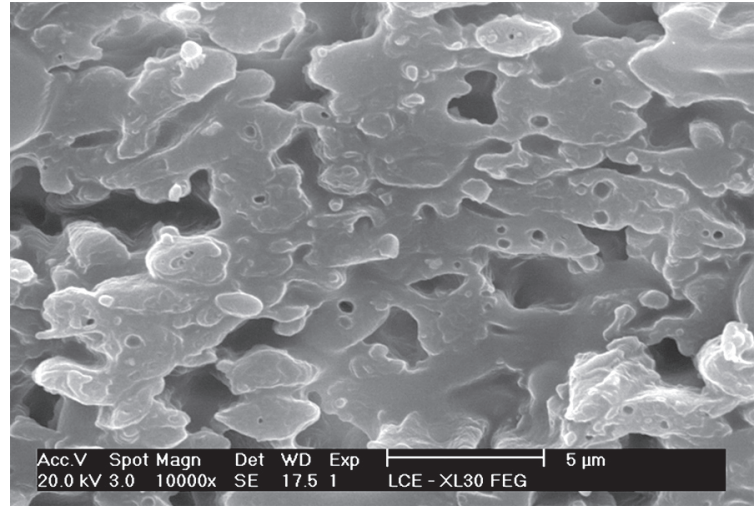

(e)

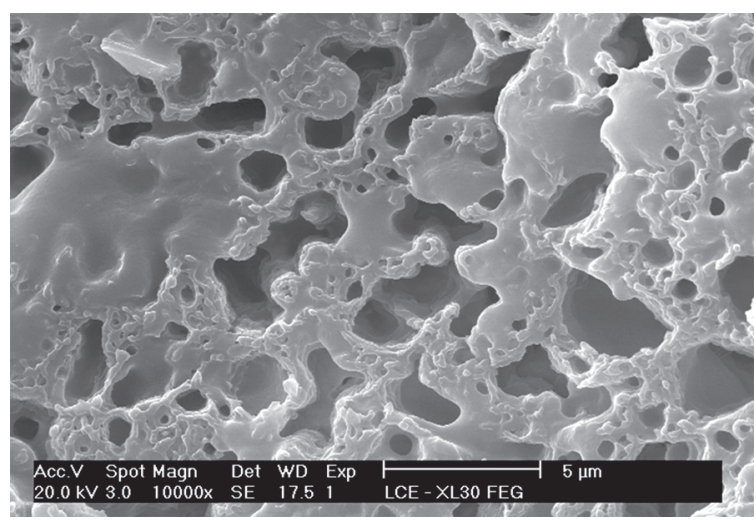

(f)

Figura 2. Micrografias das amostras HDPE/HIPS/7,5\% de compatibilizante a) 80/20/SBS; b) 80/20/SEBS; c) 60/40/SBS; d) 60/40/SEBS; e) 40/60/SBS; e f) $40 / 60 / \mathrm{SEBS}$. Barra dimensional $=5 \mu \mathrm{m}$. 
brosa da não compatibilizada, tendo-se observado uma massa disforme, conforme mostra a Figura 3. É notável a diferença na morfologia, ao comparar as micrografias das blendas com e sem adição de compatilizante, Figuras 3 a e 3 b.

Wu et al. ${ }^{[20]}$ verificaram que para a blenda compatibilizada HDPE/PS/SBS 18/72/10, apesar de uma morfologia bastante irregular, ainda era visível uma estrutura tipo fibrosa de "cordões e contas" após extração de PS e SBS com tolueno por 48 horas. No presente trabalho, houve dificuldade de manuseio dessas amostras após a extração seletiva, tendo-se conseguido caracterizar somente a morfologia de uma pequena amostra compatibilizada com SEBS, a qual está apresentada na Figura 3, comparativamente com à da correspondente não compatibilizada. Isto foi atribuído ao fato de a fase majoritária sendo HIPS, muito provavelmente a dissolução deste e dos copolímeros com tolueno tenha sido responsável por um colapso entre os domínios de HDPE, principalmente devido às maiores interações.

Quanto às propriedades térmicas analisadas, os resultados estão apresentados na Tabela 1. Com a adição dos dois tipos de compatibilizantes, existe uma diminuição da temperatura de fusão $\left(\mathrm{T}_{\mathrm{m}}\right)$ do HDPE, em qualquer proporção que estejam os componentes HDPE e HIPS. As diminuições em $\mathrm{T}_{\mathrm{m}}$ cau-

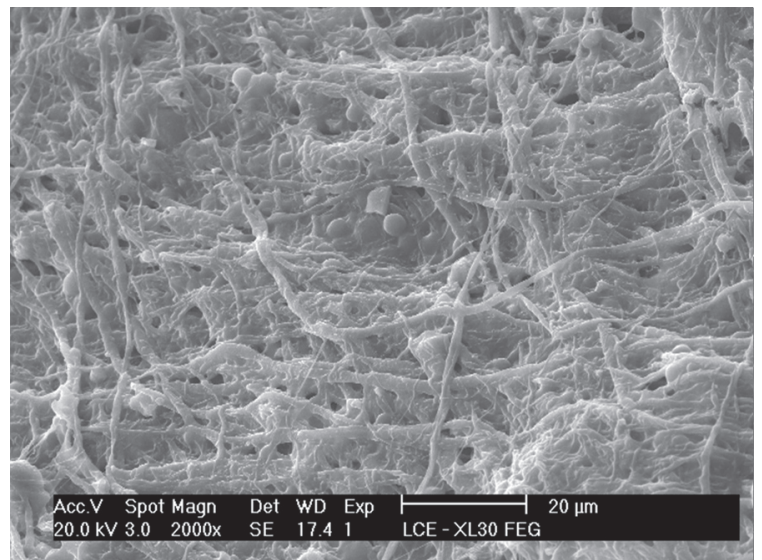

(a)

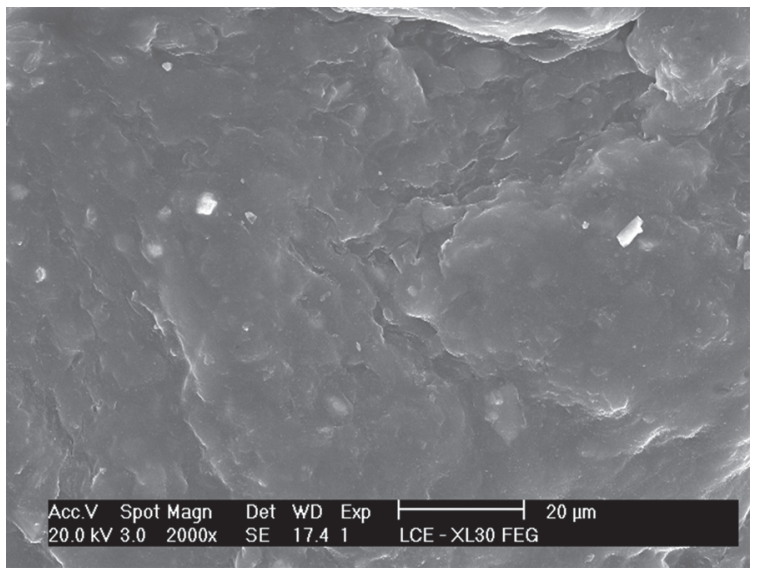

(b)

Figura 3. Micrografias das amostras HDPE/HIPS 20/80: a) sem copolímero; e b) com SEBS, Barra dimensional $=20 \mu \mathrm{m}$. (2.000x).
Tabela 1. Resultados de temperaturas de fusão $\left(\mathrm{T}_{\mathrm{m}}\right)$ e de transição vítrea $\left(\mathrm{T}_{\mathrm{g}}\right)$, entalpia de fusão $(\Delta \mathrm{H})$ e grau de cristalinidade $\left(x_{\mathrm{c}}\right)$ para as blendas sem e com adição dos copolímeros.

\begin{tabular}{cccccc}
\hline $\begin{array}{c}\text { HDPE/ } \\
\text { HIPS }\end{array}$ & $\begin{array}{c}\text { Copolímero } \\
\mathbf{( 7 , 5 \% )}\end{array}$ & $\mathbf{T}_{\mathbf{m}}\left({ }^{\circ} \mathbf{C}\right)$ & $\Delta \mathbf{H}\left(\mathbf{J} \cdot \mathbf{g}^{-1}\right)$ & $x_{\mathbf{c}}(\boldsymbol{\%})$ & $\mathbf{T}_{\mathbf{g}}\left({ }^{\circ} \mathbf{C}\right)$ \\
\hline \multirow{2}{*}{$100 / 0$} & & 134,3 & 189,4 & 64,6 & - \\
& SBS & 133,3 & 178,1 & 60,8 & - \\
& SEBS & 133,1 & 174,3 & 59,5 & - \\
\hline \multirow{2}{*}{$80 / 20$} & & 133,7 & 190,6 & 65,0 & 106,4 \\
& SBS & 133,1 & 170,2 & 58,1 & 106,5 \\
& SEBS & 133,1 & 165,8 & 56,6 & 107,9 \\
\hline \multirow{2}{*}{$60 / 40$} & & 134,4 & 189,8 & 64,8 & 105,4 \\
& SBS & 132,3 & 167,9 & 57,3 & 104,1 \\
& SEBS & 133,8 & 158,3 & 54,0 & 104,9 \\
\hline \multirow{2}{*}{$40 / 60$} & SBS & 134,0 & 189,9 & 64,8 & 104,3 \\
& SEBS & 133,1 & 206,0 & 70,3 & 102,3 \\
\hline \multirow{2}{*}{$20 / 80$} & & 132,8 & 174,5 & 59,6 & 103,3 \\
\hline & SBS & 131,9 & 190,6 & 65,0 & 101,7 \\
& SEBS & 131,7 & 162,4 & 55,4 & 103,0 \\
\hline \multirow{2}{*}{$0 / 100$} & SBS & - & - & - & 97,8 \\
& SEBS & - & - & - & 99,6 \\
\hline
\end{tabular}

sadas pelos compatibilizantes são muito próximas quando analisamos as amostras de mesma composição. Isso pode ser visualizado na Figura 4, onde se mostra uma única exceção para HDPE/HIPS 60/40.

As diminuições em $\mathrm{T}_{\mathrm{m}}$ foram interpretadas como resultado de uma diluição maior de HDPE ocasionada pela fração de moléculas de compatibilizante que se dispersaram mais amplamente nos componentes individuais da blenda, somadas às que se posicionaram na interface. Isso proporciona uma maior movimentação molecular da fase cristalina HDPE, acarretando em cristais menos perfeitos e em menor grau de cristalinidade $x_{\mathrm{c}}$, atingindo portanto mais facilmente a fusão ${ }^{[16]}$. A diminuição em $x_{c}$ foi comprovada para quase todas as amostras contendo copolímero, conforme os resul-

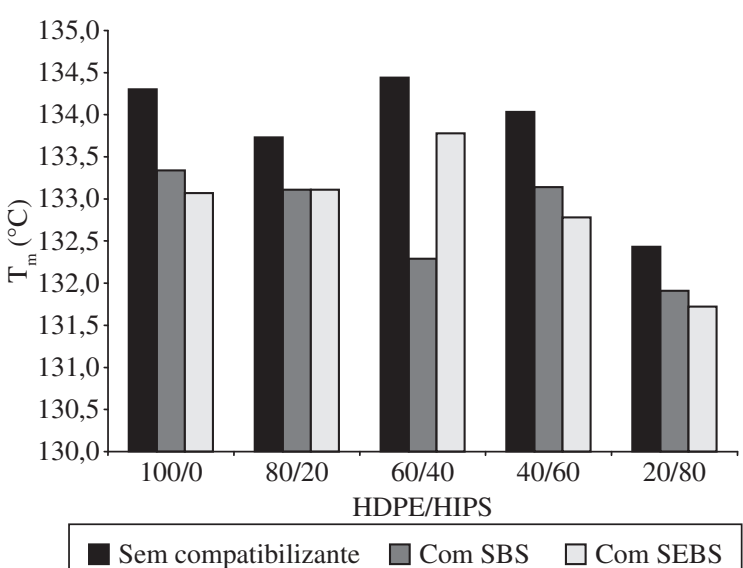

Figura 4. Influência da composição e dos copolímeros em bloco na $T_{m}$ da fase HDPE em blendas HDPE/HIPS pós-consumo. 
tados mostrados na tabela. No que diz respeito ao processamento desses materiais, a diminuição em $\mathrm{T}_{\mathrm{m}}$ e $x_{\mathrm{c}}$, mesmo que pequena, pode significar redução associada da energia despendida no processo, representando então redução dos custos de produção.

Ao comparar amostras com diferentes quantidades de HIPS, é possível verificar uma tendência de diminuição do valor da $\mathrm{T}_{\mathrm{m}}$ quando do aumento da proporção desse componente, como mostrado na Figura 4. Em termos comparativos, com SEBS conseguem-se diminuições mais significativas em $\mathrm{T}_{\mathrm{m}}$ relativamente a SBS, o que torna o copolímero tribloco mais atrativo para uso como surfactante das blendas estudadas.

Na Figura 5 é mostrado como variam os valores do calor de fusão em função da composição da amostra. Pode-se notar que, à exceção somente de HDPE/HIPS 20/80 e 40/60 contendo SBS, em todas as demais amostras a entalpia de fusão $(\Delta \mathrm{H})$, e portanto o grau de cristalinidade $x_{\mathrm{c}}$, diminui com a adição de SEBS ou SBS.

Ainda se referindo ao mesmo gráfico, fica evidente que a maior diminuição dos valores de calor de fusão está, em todos os casos, relacionada com a adição de SEBS. Isso corrobora com os resultados de $\mathrm{T}_{\mathrm{m}}$, confirmando a possível minimização de energia no processamento com SEBS. Esses resultados corroboram também com os apresentados na Figura 2, que mostram a possível existência de maior interação entre as respectivas fases compatíveis do SEBS e das misturas HDPE/HIPS.

Para analisar a variação de temperatura de transição vítrea $\mathrm{T}_{\mathrm{g}}$ (Figura 6), é importante lembrar que na faixa de temperatura estudada, a transição detectada corresponde à $\mathrm{T}_{\mathrm{g}} \mathrm{da}$ fase estirênica do HIPS, o qual é constituído por uma matriz de poliestireno com uma fase elastomérica tenacificante de polibutadieno. Uma possível pequena contribuição em $T_{\mathrm{g}} \mathrm{da}$ fase PS dos copolímeros não foi considerada nesta análise, por ser a sua concentração invariável.

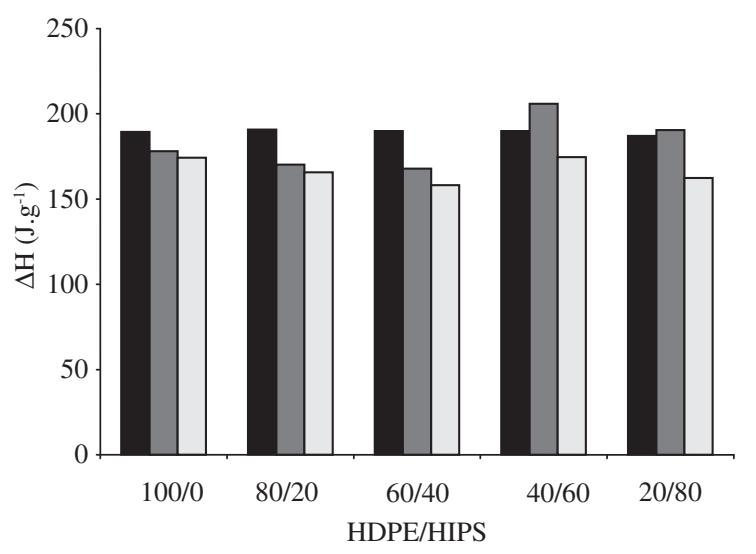

- Sem compatibilizante $\quad$ CCom SBS $\square$ Com SEBS

Figura 5. Entalpia de fusão da fase cristalina HDPE em função da composição e da adição dos copolímeros SBS e SEBS em blendas HDPE/HIPS pós-consumo.

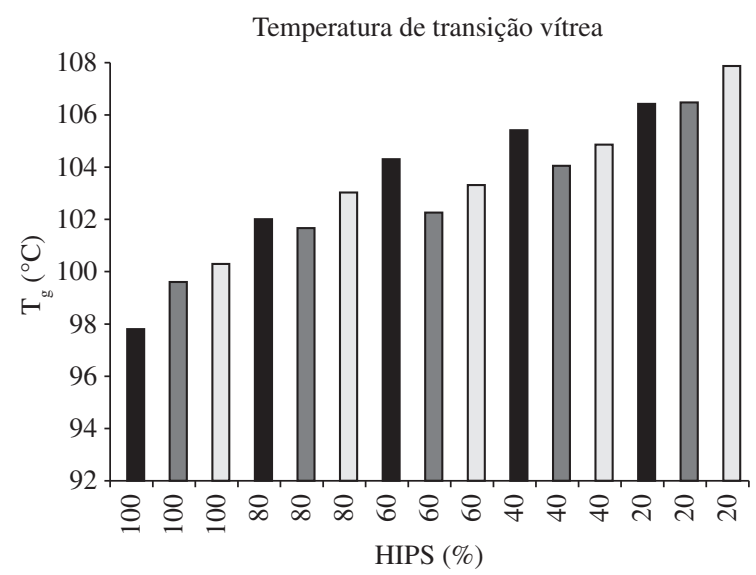

- Sem compatibilizante $\square$ Com SBS $\square$ Com SEBS

Figura 6. Variação de $\mathrm{T}_{\mathrm{g}}$ da fase estirênica do HIPS e das blendas em função da composição e da adição dos copolímeros em bloco.

A adição de 7,5\% de SBS proporciona a diminuição de $\mathrm{T}_{\mathrm{g}}$ relativamente às blendas puras, não compatibilizadas, diferentemente da adição de SEBS, em que a diminuição ocorreu somente para as composições intermediárias 40/60 e 60/40. Corrêa e Manrich ${ }^{[19]}$ verificaram também diminuições em $\mathrm{T}_{\mathrm{m}}$ e em $\mathrm{T}_{\mathrm{g}}$ ao introduzir $4,75 \%$ (5 pcr) e $9,1 \%$ (10 pcr) de SBS linear (Basf 2G 66) e o mesmo SEBS (Kraton G) em blendas de HDPE/HIPS 20/80 pós-consumo de mesma origem das utilizadas aqui. Naquele estudo, as variações em $T_{m}$ foram muito pequenas com a introdução dos copolímeros surfactantes, porém elas foram notáveis em $\mathrm{T}_{\mathrm{g}}$, com decréscimos de 4 a $10{ }^{\circ} \mathrm{C}$. Correlacionando esses resultados com a morfologia de fases, concluiu-se que o SEBS foi também mais efetivo como compatibilizante. Não se observou estrutura fibrosa em quaisquer das superfícies criofraturadas, sem extração seletiva, das amostras analisadas. As influências diferenciadas dos copolímeros, que são ambos triblocos lineares, foram devidas principalmente às diferenças nos blocos intermediários de butadieno e etileno-co-butileno. Não coube naquele estudo comparar possíveis influências devidas aos diferentes pesos moleculares das cadeias e dos segmentos individuais dos blocos por serem SBS e SEBS copolímeros diferentes.

No presente trabalho, com respeito à variação de $T_{\mathrm{g}} \mathrm{em}$ função da concentração dos componentes HDPE e HIPS, verificou-se que diminuindo a proporção de HIPS houve aumento de $\mathrm{T}_{\mathrm{g}}$, ou seja, aumentando a concentração do componente cristalino HDPE na blenda, aumentou $\mathrm{T}_{\mathrm{g}}$, independentemente da presença ou não dos copolímeros SBS e SEBS. Isso provavelmente possa ser explicado pelo fato de a temperatura de transição térmica de uma fase ser função também de características induzidas pela fase externa. A presença em maiores proporções de uma fase envolvente cristalina mais rígida, como é o caso do polietileno, restringe a movimentação molecular da fase dispersa, deslocando sua temperatura de transição vítrea para temperaturas maiores. Analisando as micrografias, verifica-se que na fase amorfa HIPS, mesmo na composição HDPE/HIPS 40/60 (Figuras 1 e 2), as dimensões 
Temperatura de transição vítrea

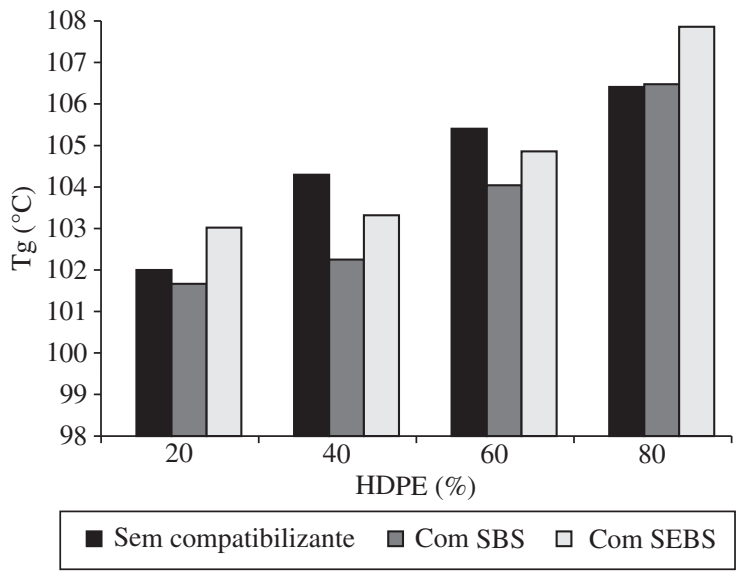

Figura 7. Influência do tipo de agente compatibilizante sobre a $T_{g}$ da fase estirênica das blendas HDPE/HIPS pós-consumo.

"transversais" são relativamente pequenas, sendo menores que $2 \mu \mathrm{m}$ na maioria das amostras. Nesse caso, seria razoável considerar que a fase estirênica possa sofrer influência da fase externa cristalina mais rígida HDPE, de forma a aumen$\operatorname{tar} \mathrm{T}_{\mathrm{g}}$ com o aumento da fração desse componente.

Os resultados de $\mathrm{T}_{\mathrm{g}}$ comparativamente entre as blendas compatibilizadas e as não compatibilizadas, como anteriormente comentado, mostraram que não há uma influência comum para SBS e SEBS em todas as composições. Com a adição de SBS a transição vítrea manteve-se igual na blenda $80 / 20$ ou deslocou-se para temperaturas menores em todas as demais blendas e a adição de SEBS provocou diminuição em $\mathrm{T}_{\mathrm{g}}$ para as composições 60/40 e 40/60, porém aumento para as demais $80 / 20$ e 20/80, quando comparadas com as respectivas misturas sem copolímero.

Por outro lado, foi interessante observar os efeitos provocados na temperatura de transição vítrea pela adição de SBS e de SEBS, quando comparados entre si nas mesmas composições, como bem visualizado na Figura 7. Em todos os casos, a $\mathrm{T}_{\mathrm{g}}$ da fase PS é maior na presença de SEBS do que na presença de SBS, comprovando que há de fato diferenças significativas de atuação como agente interfacial entre as fases HDPE e HIPS, devidas às diferenças nos comonômeros e nas estruturas multibloco e linear.

\section{Conclusões}

$\mathrm{Na}$ análise morfológica, para as blendas que não foram compatibilizadas, como era esperado foi possível observar interfaces lisas e "limpas", o que evidenciou a inexistência de ligações fortes entre HIPS e HDPE. A formação de fibras bastante longas igualmente lisas de HDPE na matriz de HIPS, para a composição HDPE/HIPS 20/80, comprovou a incompatibilidade entre as fases. A adição tanto de SBS quanto de SEBS ocasionou uma diminuição do tamanho médio dos domínios da fase minoritária, além de maior rugosidade tanto na superfície de fratura quanto nas cavidades deixadas pela extração do HIPS e dos copolímeros, o que mostrou a interação entre as fases.

Quanto ao tipo de compatibilizante utilizado, foi possível observar que a adição de SEBS proporcionou aos domínios da fase dispersa tamanhos menores ainda e melhor distribuídos quando comparado com SBS multibloco. A interpenetração entre as fases mais favorecida com SEBS ficou evidente na morfologia considerada mais semelhante à co-contínua apresentada pelas blendas HDPE/HIPS 40/60 compatibilizadas. A morfologia "colapsada" após a extração da matriz nas composições com 20\% de HDPE contendo copolímeros foi atribuída também à interação interfases proporcionada pelos modificadores interfaciais.

Os compatibilizantes foram ainda responsáveis por uma diminuição da $T_{m}$ do HDPE para todas as composições estudadas, tendo-se considerado esse fato como resultado de uma maior diluição provocada pelas moléculas de compatibilizante e de HIPS. Aumentando a quantidade de HIPS, diminuiu $\mathrm{T}_{\mathrm{m}}$, e ao se adicionar compatibilizante, principalmente SEBS, essa diminuição foi ainda maior. $\mathrm{O}$ calor de fusão $\Delta \mathrm{H}$ e o grau de cristalinidade $x_{\mathrm{c}}$ diminuíram com a adição de SEBS novamente para todas as composições, o que não foi observado quando o SBS foi adicionado. Para as amostras contendo SBS, os valores de $\Delta \mathrm{H}$ e $x_{\mathrm{c}}$ diminuíram somente para as composições em que o HDPE constituiu a fase matriz.

Aumentando-se a quantidade de polietileno da blenda, aumentou a $T_{g}$ da fase estirênica do HIPS. Esse fato foi atribuído a características induzidas pela fase envolvente cristalina mais rígida HDPE, cuja influência tornou-se mais efetiva com o aumento de sua fração mássica. Em todos os casos, as blendas contendo SEBS apresentaram maior $\mathrm{T}_{\mathrm{g}}$ do que aquelas contendo SBS, tendo-se concluído que uma provável maior facilidade de a estrutura linear do copolímero tribloco linear em interagir e até se misturar simultaneamente às diferentes fases tenha contribuído para uma compatibilização mais efetiva das blendas HDPE/HIPS pós-consumo.

\section{Agradecimentos}

Os autores agradecem à FAPESP pelo apoio financeiro e pela bolsa de estudos concedida e à BASF pela doação de material.

\section{Referências Bibliográficas}

1. http://www.cempre.org.br, acesso em 17/10/2007.

2. Manrich, S. \& Santos, A. S. F. e - "An Overview of Recent Advances and Trends in Plastic Recycling. in: Conservation and Recycling of Resources: New Research, cap.1, Christian V. Loeffe (ed.), Nova Science Publishers, Hauppauge, NY (2006).

3. Lazo, N. D. B. \& Scott, C. E. - Polymer, 40, p.5469 (1999).

4. Wang, Y.; Xiao, Y.; Zhang, Q.; Gao, X-L. \& Fu, Q. Polymer, 44, p.1469 (2003). 
5. Smit, I. Radonjic, G. \& Hlavatá, D. - Eur. Polym. J., 40, p.1433 (2004).

6. Pospísil, J.; Fortelný, I.; Michálková, D.; Krulis, Z. \& Slouf, M. - Polym. Degrad. Stab., 90, p.244 (2005).

7. Li, J. \& Favis, B. D. - Polymer, 42, p.5047 (2001).

8. Galloway, J. A.; Jeon, H. K.; Bell, J. R. \& Macosko, C. W. - Polymer, 46, p.183 (2005).

9. Willemse, R. C.; Speijer, A.; Langeraar, A. E. \& Boer, P. - Polymer, 40, p.6645 (1999).

10. Luzinov, I.; Xi, K.; Pagnoulle, C.; Huynh-Ba, G. \& Jérôme, R. - Polymer, 40, p.2511 (1999).

11. Pascu, M.; Vasile, C. \& Gheorghiu, M. - Mat. Chem. Phys. 80, p.548 (2003).

12. Halimatudahliana; Ismail, H. \& Nasir, M. - Polym. Test., 21, p.163 (2002).

13. Mélo, T. J. A.; Carvalho, L. H.; Calumby, R. B.; Brito, K. G. Q.; D’Almeida, J. R. M. \& Spieth, E. - Polímeros: Ciência e Tecnologia, 10, p.82 (2000).
14. Faravelli, T.; Bozzano, G.; Colombo, M.; Ranzi, E. \& Dente, M. - J. Anal. Appl. Pyrolysis, 70, p.761 (2003).

15. Tao, Y.; Lebovitz, A. H. \& Torkelson, J. M. - Polymer, 46, p.4753 (2005).

16. Chen, B.; Li, X.; Xu, S.; Tang T.; Zhou, B. \& Huang, B. - Polymer, 43, p.953 (2002).

17. Danella Jr, O. J. \& Manrich, S. - Polym. Sci., 45, p.1086 (2003).

18. Santana, R. M. C. \& Manrich, S. - J. Appl. Polym. Sci., 87, p.747 (2003).

19. Corrêa, A. C. \& Manrich, S. - "Compatibilization of post consumer high impact polystyrene (HIPS)/high density polystyrene (HDPE) blends", in: Anais do X International Macromolecular Colloquium, Gramado - RS, Abr (2005).

20. Wu, J.; Guo, B.; Chan, C-M.; Li, J. \& Tang, H-S. - Polymer, 42, p.8857 (2001).

Enviado: $25 / 10 / 07$

Reenviado: $26 / 12 / 07$

Aceito: $21 / 01 / 08$ 